\title{
Bulk Tank Somatic Cell Counts Analyzed by Statistical Process Control Tools to Identify and Monitor Subclinical Mastitis Incidence
}

\author{
J. M. Lukas, ${ }^{1}$ D. M. Hawkins ${ }^{2}$, M. L. Kinsel, ${ }^{3}$ and J. K. Reneau ${ }^{1}$ \\ ${ }^{1}$ Department of Animal Science, and \\ ${ }^{2}$ School of Statistics, University of Minnesota, St. Paul 55108 \\ ${ }^{3}$ Agricultural Information Management, Inc., Ellensburg, WA 98926
}

\begin{abstract}
The objective of this study was to examine the relationship between monthly Dairy Herd Improvement (DHI) subclinical mastitis and new infection rate estimates and daily bulk tank somatic cell count (SCC) summarized by statistical process control tools. Dairy Herd Improvement Association test-day subclinical mastitis and new infection rate estimates along with daily or every other day bulk tank SCC data were collected for 12 mo of 2003 from 275 Upper Midwest dairy herds. Herds were divided into 5 herd production categories. A linear score [LNS $=\ln (\mathrm{BTSCC} / 100,000) /$ $0.693147+3$ ] was calculated for each individual bulk tank SCC. For both the raw SCC and the transformed data, the mean and sigma were calculated using the statistical quality control individual measurement and moving range chart procedure of Statistical Analysis System. One hundred eighty-three herds of the 275 herds from the study data set were then randomly selected and the raw (method 1) and transformed (method 2) bulk tank SCC mean and sigma were used to develop models for predicting subclinical mastitis and new infection rate estimates. Herd production category was also included in all models as 5 dummy variables. Models were validated by calculating estimates of subclinical mastitis and new infection rates for the remaining 92 herds and plotting them against observed values of each of the dependents. Only herd production category and bulk tank SCC mean were significant and remained in the final models. High $\mathrm{R}^{2}$ values $(0.83$ and 0.81 for methods 1 and 2 , respectively) indicated a strong correlation between the bulk tank SCC and herd's subclinical mastitis prevalence. The standard errors of the estimate were 4.02 and $4.28 \%$ for methods 1 and 2, respectively, and decreased with increasing herd production. As a case study, Shewhart Individual Measurement Charts were plotted from the bulk tank SCC to identify
\end{abstract}

\footnotetext{
Received December 27, 2004.

Accepted July 9, 2005.

Corresponding author: Jeffrey K. Reneau; e-mail: renea001@ umn.edu.
}

shifts in mastitis incidence. Four of 5 charts examined signaled a change in bulk tank SCC before the DHI test day identified the change in subclinical mastitis prevalence. It can be concluded that applying statistical process control tools to daily bulk tank SCC can be used to estimate subclinical mastitis prevalence in the herd and observe for change in the subclinical mastitis status. Single DHI test day estimates of new infection rate were insufficient to accurately describe its dynamics.

(Key words: bulk tank somatic cell count, mastitis prevalence, statistical process control)

Abbreviation key: BTSCC = bulk tank SCC, Cpk = capability index, HPC = herd production category, LNS = linear score, LNSmean = linear score mean, LNSsigma = linear score sigma, NIR = new infection rate, $\mathbf{N I R e}=$ estimated new infection rate, $\mathbf{S C C m e a n}=$ SCC mean, SCCsigma = SCC sigma, $\mathbf{S M}=$ subclinical mastitis prevalence, $\mathbf{S M e}=$ estimated subclinical mastitis prevalence, $\mathbf{S P C}=$ statistical process control.

\section{INTRODUCTION}

Currently, subclinical mastitis prevalence (SM) and new infection rate (NIR) are estimates derived from individual cow monthly DHI testing. The values obtained are therefore representative of the state of the herd on one particular day of the month when the test was performed and are only available to the herds that are enrolled in DHI or similar programs. At present, only $46 \%$ of the herds in the United States participate in the DHI record system (Wiggans, 2004), whereas every herd selling milk will have routine component and SCC testing done most likely once a week. In the Upper Midwest, these bulk tank tests are completed on every milk pick up. Herds' SM and NIR estimates are important for mastitis control. The estimates of SM and NIR provide feedback on the impact of milk quality improvement efforts on individual herd, state, or national levels. At present, they are limited to single monthly estimates available for less than half of the herds in the United States. More frequent measures of SM and NIR would provide dairy managers and their consultants with more timely awareness of emerging 
mastitis trends; however, the expense and disruption of daily sampling of individual cows is both cost prohibitive and impractical. The bulk tank SCC (BTSCC) depends on SM and NIR among cows being milked into the bulk tank (Eberhart et al., 1982; Harmon, 1994). Observing the BTSCC is a readily available and inexpensive way of monitoring the mastitis trend in the herd. Recently, researchers have suggested monitoring the BTSCC with the use of statistical process control (SPC) charts (Marsh et al., 1997; Reneau and Kinsel, 2001). Statistical process control is a quality improvement tool that has been extensively used in nonagricultural industries in the past century. In the last $25 \mathrm{yr}$, attempts have been made to apply SPC in swine (Sard, 1979; Dial et al., 1996), beef and poultry (Cowen et al., 1994; Fernandez, 1995) and dairy (De Vries and Conlin, 2003). The statistical methods and run rules applied to control charts aim to distinguish (with a certain level of confidence) between common cause variation due to usual day-to-day fluctuations in process output and variation due to special cause such as changes in the materials, people, machine, environment, or method in which the process was performed. This distinction is meant to aid in identifying when a true change has occurred and action is needed to assure desired process performance. The simplicity of the charts makes them easy to use not only by management but also by foremen or production line workers who have a direct impact on the process (Ishikawa, 1984). These characteristics of control charts have contributed to their successful implementation in nonagricultural industry.

The interest in application of SPC charts in dairy production systems is increasing (Kinsel, 2004). For many herds, SPC charts along with a current BTSCC mean and sigma are being automatically computed from each bulk tank pickup test result (Kinsel, 2004) and provided as a service (via the internet) to the dairy managers and dairy consultants. Using the MilkLab internet system (www.dairyperformance.com), an increasing number of dairy managers from the Upper Midwest monitor their results daily for changing BTSCC trends, and use the SPC charts to determine whether their milk quality management is getting better or worse (special cause variation) or staying the same (common cause variation). Herd managers using the MilkLab service have daily access not only to SCC charts but also to current SCC mean and sigma, making the use of other SPC tools possible.

One of the tools of SPC is the capability index (Cpk) used to summarize process performance. The capability index is calculated by subtracting the process average output from a desired standard or specification and dividing the difference by 3 sigmas of the output. It is used to measure the capability of the process to meet a specific quality standard. Scientists have repeatedly researched the relationship between BTSCC and mastitis prevalence in the herd and have reported different results (Emanuelson and Funke, 1991). Only recently has the application of SPC tools in agriculture prompted attempts to summarize the performance of the milk production processes in the form of a Cpk index and relate it to SM in the herd (Niza-Ribeiro et al., 2004). Although those researchers were able to show high correlation coefficients between BTSCC and SM, the models developed in the study estimated SM from herds BTSCC with an average $8.8 \%$ error. In our study, we attempted to identify the relationship between the components of the Cpk index (mean and variation) calculated from the BTSCC and the DHIA SM and NIR estimates for the herd. We also used more frequent BTSCC testing than was utilized in previous research, and included herd production as a covariate in our models.

In view of the growing interest in SPC by the livestock industry, it can be expected that its use and application will continue to spread. Further study of the relationship between BTSCC summarized by SPC tools and mastitis incidence in the herd is therefore not only promising, but also necessary, in anticipation of the more widespread use of SPC on the dairy farm and in the dairy industry.

The objective of this study was to examine the relationship between monthly DHI SM and NIR estimates and the daily BTSCC monitored and summarized by SPC tools for 275 Minnesota dairies. The specific aim of this research was to determine if there was sufficiently strong correlation between the BTSCC average and standard deviation included in the SPC Cpk index calculation and SM and NIR for estimates to provide easy to obtain and accurate information for milk quality management decisions. Such a value-added use of bulk tank SCC data already being collected could offer a reliable and economical means of providing SM and NIR estimates for all US dairies.

\section{MATERIALS AND METHODS}

Our study data set contained BTSCC data as well as SM and NIR estimates for 275 herds.

\section{BTSCC Data}

The BTSCC were collected daily or every other day for 12 mo (January though December 2003) from 275 Upper Midwest dairies. A linear score [LNS = $\ln (\mathrm{BTSCC} / 100,000) / 0.693147+3])$ was calculated from the raw BTSCC data. Both the raw SCC and the LNS data were kept in the data set for further analysis. The 
Statistical Quality Control Individual Measurement and Moving Range Chart procedure of SAS (SAS Institute, Inc., Cary, NC) was run separately for the SCC and LNS data, by herd and month. The mean SCC, LNS, and sigma, estimated from the average moving range of size 2 ( $\operatorname{sigma}=\mathrm{R} / \mathrm{d}_{2}$ ), of SCC and LNS were calculated for each herd for each of the $12 \mathrm{mo}$. The yearly mean and sigma for SCC (SCCmean and SCCsigma) and LNS (LNSmean and LNSsigma) were then calculated for each herd from the monthly values.

\section{SM and NIR Estimates}

Subclinical mastitis prevalence and NIR for 275 herds were obtained for each day the herd was tested by the Minnesota DHI program in 2003. Number of test days ranged from 2 to 12 with $82 \%$ of the herds having 10 or more test days, $17 \%$ of the herds having between 5 and 9 test days, and the remaining $1 \%$ of the herds having 4 or fewer test days.

Subclinical mastitis prevalence was determined for each herd from the number of cows that had an SCC above 200,000 on any of the DHI test days divided by the total number of cows tested during the 12-mo period. New infection was defined as: 1) those cows that had an SCC greater than 200,000 cells $/ \mathrm{mL}$ on the first DHI test after calving, or 2) those with SCC less than 200,000 cells $/ \mathrm{mL}$ in the previous month, but greater than 200,000 cells $/ \mathrm{mL}$ in the present month. Values obtained for each test day were added for each herd and divided by the total number of cows tested during the 12-mo period to obtain the yearly average NIR for each herd.

\section{Herd Production}

The 275 herds were divided into 5 herd production categories (HPC) based on the average amount of milk shipped each month in 2003 (USDA classification). Herd production categories were defined as follows: 1 ) included all herds with an average amount of milk shipped less than $23,000 \mathrm{~kg}(50,000 \mathrm{lb}), 2)$ between $23,000 \mathrm{~kg}(50,000 \mathrm{lb})$ and $45,000 \mathrm{~kg}(100,000 \mathrm{lb}), 3)$ between $45,000 \mathrm{~kg}(100,000 \mathrm{lb})$ and $90,000 \mathrm{~kg}(200,000$ $\mathrm{lb}), 4)$ between $90,000 \mathrm{~kg}(200,000 \mathrm{lb})$ and $227,000 \mathrm{~kg}$ $(500,000 \mathrm{lb})$, and 5) greater than $227,000 \mathrm{~kg}(500,000$ lb). Assuming an average of $30 \mathrm{~kg} / \mathrm{cow}$ per d, HPC 1 , $2,3,4$, and 5 corresponded to fewer then 25 cows, from 25 to 49 cows, from 50 to 99 cows, from 100 to 249 cows, and 250 or more cows, respectively.

The herds in the data set had a herd size distribution similar to the one reported in Minnesota in 2001 (USDA, 2003). Forty-two percent of herds had fewer than 50 cows (vs. 49\% in Minnesota), 38\% had between 50 and 99 head (vs. 38\% in Minnesota), 9\% of herds were in the 100 to 199 range (vs. 9\% in Minnesota), and $11 \%$ of herds had 200 or more cows (vs. $4 \%$ in Minnesota). Based on monthly DHIA reports in 2003, the herds in the study data set averaged 386,966 SCC (SD 194,350) with a 9041-kg (SD: $1564 \mathrm{~kg}$ ) rolling herd average, $34.7 \%$ (SD 12.4\%) SM, and $12.6 \%$ (SD 6.1\%) NIR.

\section{Developing the Models}

Two methods were used to determine the relationship between a herd's SM and NIR and its BTSCC. In each method, 183 of the 275 herds from the study data set were randomly selected and used to develop separate models for SM and NIR using the SAS GLM procedure. Herd production category was entered as 5 dummy variables with BTSCC mean and sigma used as the quantitative predictors in all models. A full model including all 2- and 3-way interactions was fit. Insignificant terms were then removed from the model based on the $P>$ 0.05 criterion.

In method 1, the 2 herd-health characteristics (SM and NIR) were related to the BTSCC through means and sigmas calculated from the raw BTSCC (SCCmean and SCCsigma). Method 2 used the means and sigmas obtained from the transformed data (LNSmean and LNSsigma) to describe the same relationships.

\section{Model Validation}

To validate the developed models, SM and NIR values observed for the remaining 92 of the herds were compared with the estimated subclinical mastitis values (SMe) and new infection rates (NIRe) calculated from the linear regression models developed. The estimated values of SMe and NIRe were then plotted against the observed SM and NIR values. The $R^{2}$ value for each model and a corresponding standard error of estimation $\left(\sigma_{\text {est }}\right)$ for each of the models, and for each herd production category within each model, were calculated using the following formulas:

$$
\begin{gathered}
\sigma_{\text {est }}=\sqrt{ }\left(\Sigma\left(\hat{\mathrm{y}}_{\mathrm{i}}-\mathrm{y}_{\mathrm{i}}\right)^{2} / \mathrm{n}\right) \\
\mathrm{R}^{2}=1-\Sigma\left(\hat{\mathrm{y}}_{\mathrm{i}}-\mathrm{y}_{\mathrm{i}}\right)^{2} / \Sigma\left(\tilde{\mathrm{y}}-\mathrm{y}_{\mathrm{i}}\right)^{2}
\end{gathered}
$$

where $\hat{y}$ is the estimated value of SMe or NIRe, $y$ is the observed value of SM or NIR, y is the average SM or NIR of the observed values, and $\mathrm{n}$ is the total number of observations used for validation of the model. 
Table 1. Change in subclinical mastitis prevalence (SM) between 2 adjacent DHI test days marking the greatest increase in subclinical mastitis (D1 and D2), and mean and sigma (estimated from the average moving range) of the bulk tank SCC from the $30 \mathrm{~d}$ preceding D1 of the 5 herds used in the case study.

\begin{tabular}{llllr}
\hline \multirow{2}{*}{$\begin{array}{l}\text { Herd } \\
\text { production } \\
\text { category }\end{array}$} & Milk yield, kg & $\begin{array}{l}\text { Change } \\
\text { in SM, } \%\end{array}$ & SPC chart \\
\cline { 3 - 5 } & $<23,000$ & 10 & Mean & Sigma \\
\hline 1 & 23,000 to 45,000 & 33 & 575 & 181 \\
3 & 45,000 to 90,000 & 22 & 333 & 71 \\
4 & 90,000 to 227,000 & 16 & 362 & 99 \\
5 & $\geq 227,000$ & 13 & 311 & 81 \\
\hline
\end{tabular}

\section{Case Study}

To illustrate the use of SPC charts to detect changes in subclinical mastitis from the 92 validation herds for each production category, 1 herd was chosen that experienced the greatest change in subclinical mastitis in 2003. All 5 herds had bulk tank milk picked up and tested every other day. All BTSCC results obtained between the 2 adjacent DHIA test days (D1 and D2) marking the largest shift in subclinical mastitis prevalence and the $30 \mathrm{~d}$ preceding D1 for each of the 5 herds were plotted on a Shewhart Individual Measurement chart. The mean and sigma (estimated by the average moving range) used to calculate the center line (estimated by the mean) and upper and lower 3 sigma control limits (mean $\pm 3 \times \mathrm{R} / \mathrm{d}_{2}$ ) were obtained from results from the $30 \mathrm{~d}$ preceding $\mathrm{D} 1$. These limits along with the following run rules were applied to the Shewhart Individual Measurement charts and used to identify signals of change in BTSCC between D1 and D2. The run rules were: $\mathrm{A}=\mathrm{A}$ point outside the 3 -sigma control limits; $\mathrm{B}=9$ points in a row on one side of the center line; $\mathrm{C}=6$ points in a row, either all increasing or all decreasing; $\mathrm{D}=2$ out of 3 points in a row in zone $\mathrm{A}$ or beyond on one side of the center line; and $\mathrm{E}=4$ out of 5 points in a row in zone $B$ or beyond on one side of the center line.

The change in subclinical mastitis prevalence between D1 and D2 ranged from $10 \%$ for herd A (HPC 1) to $33 \%$ for herd B (HPC 2). The mean SCC during the same period ranged from 311,000 for herd E (HPC 5) to 575,000 for herd A (HPC 1), whereas the variation ranged from 45,000 to 181,000 SCC (Table 1). The interval between D1 and D2 differed between herds and lasted 28, 53, 34, 29, and $35 \mathrm{~d}$ for herds A through $\mathrm{E}$, respectively.

\section{RESULTS AND DISCUSSION}

\section{Subclinical Mastitis Prevalence}

The models resulting from the 2 methods are presented in Table 2 . In both methods, the standard devia- tion and all interactions were removed based on the $P$ $>0.05$ criterion. This suggests that when the average SCC is included in the model, the SCC sigma does not significantly improve the determination of SM in the herd. A recent study of the relationship between BTSCC and SM in the herd (Niza-Ribeiro et al., 2004) has reported similar results. Those researchers concluded that SM is more dependent on the BTSCC mean than on the variation in BTSCC. Both models have high $\mathrm{R}^{2}$ values, meaning that, regardless of the method used to obtain the mean BTSCC (LNS or SCC), high correlations exist between SM and the BTSCC.

The distribution of herds' individual bulk tank SCC was positively skewed (average skewness $=0.721$ ). Linear score is a transformation designed to create a variable that is approximately normally distributed. The SCC used in this analysis however were means of individual raw (SCC) or log transformed (LNS) SCC. Therefore, the benefit of using log-transformed data as opposed to raw data was limited. Based on a comparison of the $\mathrm{R}^{2}$ values, the log transformation did not result in a significant improvement in model fit. The high $\mathrm{R}^{2}$ values also indicate that both models were successful at explaining the variation of the SM values in the data set used to develop the models.

The validation of the models is presented in Figures 1 and 2. The straight lines on the plots represent the ideal situation, in which the estimated values are equal to the observed values in the validation data set $(\mathrm{SMe}=$ $\mathrm{SM}$ ). The data in both of the plots seems equally distributed along the $\mathrm{SMe}=\mathrm{SM}$ line with no obvious patterns on either end. The standard errors of estimate by HPC (Table 3), however, indicate that for both models, the error decreases as the herd monthly production increases. This is in accordance with the results reported by Emanuelson and Funke (1991) who observed that the correlation between prevalence of mastitis in the herd and BTSCC increases as the herd monthly production increases. The problem of larger error of estimation for SM in small herds could be associated with the greater influence that one cow can have on BTSCC in a small rather than a large herd (Belisto et al., 2004). 
Table 2. Models developed by regressing subclinical mastitis prevalence (SM) against the mean and sigma (estimated from the average moving range) of bulk tank SCC (BTSCC) and herd production category (HPC). ${ }^{1}$

\begin{tabular}{|c|c|c|c|c|}
\hline Term & Estimate & $\begin{array}{l}\text { Standard } \\
\text { error }\end{array}$ & $P$ & Model $\mathrm{R}^{2}$ \\
\hline Method 1 & & & & 0.833 \\
\hline SCCmean $\times 10^{-5}$ & 0.678 & 0.024 & $* *$ & \\
\hline HPC1 & 0.129 & 0.015 & $* *$ & \\
\hline HPC2 & 0.106 & 0.011 & $* *$ & \\
\hline HPC3 & 0.098 & 0.010 & $* *$ & \\
\hline HPC4 & 0.086 & 0.012 & $* *$ & \\
\hline HPC5 & 0.079 & 0.014 & $* *$ & \\
\hline Method 2 & & & & 0.835 \\
\hline LNSmean & 0.154 & 0.006 & $* *$ & \\
\hline HPC1 & -0.322 & 0.029 & $* *$ & \\
\hline HPC2 & -0.362 & 0.027 & $* *$ & \\
\hline HPC3 & -0.376 & 0.026 & $* *$ & \\
\hline HPC4 & -0.395 & 0.027 & $* *$ & \\
\hline HPC5 & -0.407 & 0.028 & $* *$ & \\
\hline
\end{tabular}

${ }^{1}$ Method 1 used raw BTSCC mean (SCCmean) and sigma (SCCsigma) as independent variables. Method 2 used linear score mean (LNSmean) and sigma (LNSsigma). Only significant factors $(P \leq 0.05)$ were kept in the model.

$* * P \leq 0.01$

In small herds, certain management practices like the elimination of milk from cows with high SCC from the tank would significantly reduce the BTSCC, but would not be reflected in SM in the herd, because all cows in milk on the DHIA test day are used to estimate herd SM, regardless of whether they are milked into the bulk tank or not (Norman et al., 2000; Niza-Ribeiro et al., 2004). In these cases, SM estimated from the BTSCC would be lower then the actual SM in the herd.

The standard error of estimation and the $\mathrm{R}^{2}$ values are similar for both methods (Figures 1 and 2) with method 1 having slightly higher $\mathrm{R}^{2}$ values $(0.834$ vs. $0.811)$ and lower $\sigma_{\text {est }}(4.02$ vs. $4.28 \%)$. Apart from the slight superiority of the first method based on its statis-

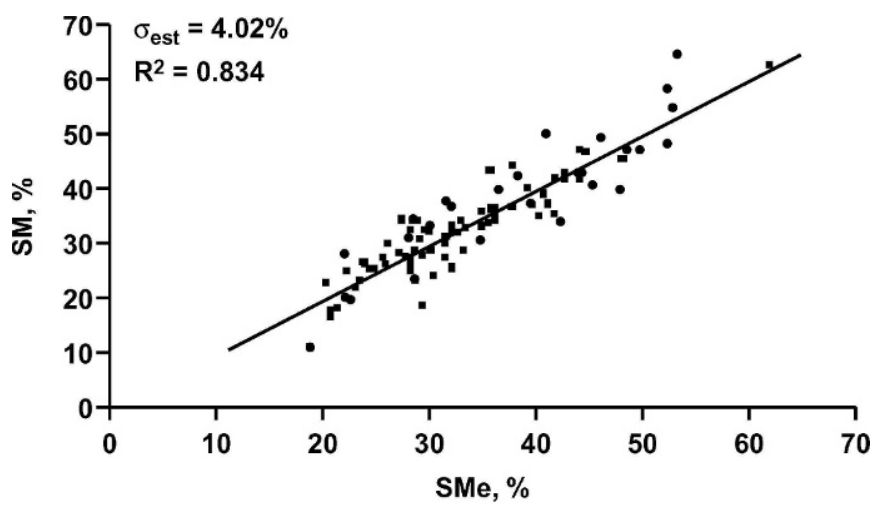

Figure 1. Validation of model 1: Plot of observed subclinical mastitis values $(\mathrm{SM})$ against the subclinical mastitis estimated (SMe) from average of raw bulk tank SCC (SCCmean) and herd production category. The straight line on the plot is defined by SM = SMe. Standard error of estimation $\left(\sigma_{\text {est }}\right)$ and $\mathrm{R}^{2}$ for model validation are given in the top left corner of the plot. tical analysis, there is another advantage of using the raw SCC to estimate SM as opposed to the LNS. Every farm that ships milk to the processor receives the BTSCC test results in the form of raw SCC. Therefore, calculating a linear score would be more troublesome and confusing than using the raw SCC with which farmers are more familiar.

The size of the standard estimation error $( \pm 4.02 \%)$ makes the confidence interval for the SM estimation very wide. It is therefore essential to identify how this kind of estimation should be used to be a helpful tool to the dairy manager or farm consultant. The estimate should not constitute the sole basis for major management decisions such as cow culling, or major bedding

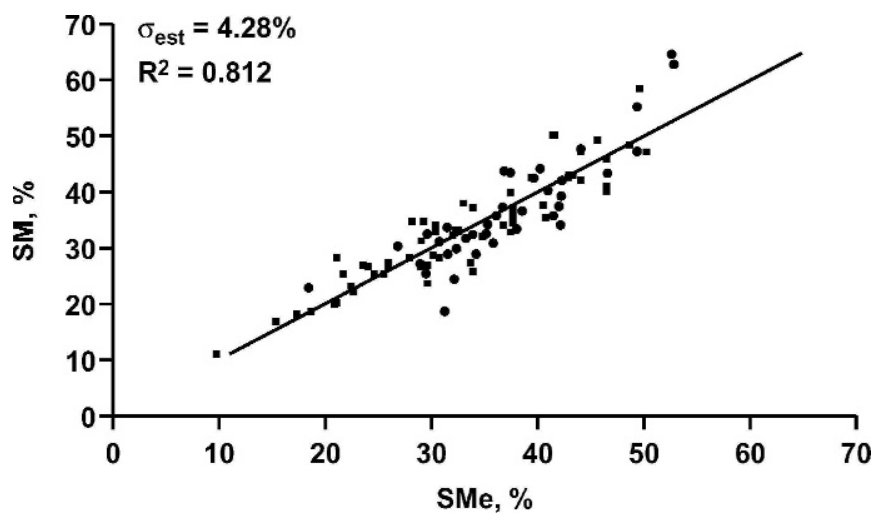

Figure 2. Validation of model 2: Plot of observed subclinical mastitis values (SM) against the subclinical mastitis estimated $(\mathrm{SMe})$ from the average of linear score of bulk tank SCC (LNSmean) and herd production category. The straight line on the plot is defined by $\mathrm{SM}=$ SMe. Standard error of estimation $\left(\sigma_{\text {est }}\right)$ and $\mathrm{R}^{2}$ for model validation are given in the top left corner of the plot. 
Table 3. Standard error of estimation of subclinical mastitis prevalence by herd production category (HPC).

\begin{tabular}{lllll}
\hline & & \multicolumn{2}{c}{$\begin{array}{c}\text { Standard error } \\
\text { of estimation, \% }\end{array}$} \\
\cline { 3 - 5 } HPC & Milk yield, kg & $\begin{array}{c}\text { No. of } \\
\text { herds }\end{array}$ & Model 1 & Model 2 \\
\hline 1 & $<23,000$ & 10 & 4.26 & 5.15 \\
2 & 23,000 to 45,000 & 26 & 4.10 & 4.65 \\
3 & 45,000 to 90,000 & 35 & 4.08 & 4.14 \\
4 & 90,000 to 227,000 & 14 & 3.92 & 3.68 \\
5 & $\geq 227,000$ & 7 & 3.22 & 4.16 \\
Total & & 92 & 4.02 & 4.28 \\
\hline
\end{tabular}

or milking system changes. Rather, it can be a starting point in attempt to monitor and control SM on the farm. On farms that are not enrolled in the DHI program, the use of this estimation gives a reliable SM estimate as opposed to no information on whole herd infection status. Estimating SM in the herd based on BTSCC can be the initial step in identifying mastitis problems in the herd and should lead to subsequent individual cow SCC testing. Observing BTSCC charts for signals of assignable cause variation can be used to monitor change in the herd infection status between test days in herds that are already on DHIA, as shown in the case study examples.

Researchers have previously attempted to relate SM in the herd to BTSCC, reporting correlation coefficients ranging from 0.46 to 0.96 (Emanuelson and Funke, 1991). More recently, Niza-Ribeiro et al. (2004) investigated the relationship between SPC Cpk index calculated from the mean and standard deviation estimates of the BTSCC and the subclinical mastitis values of the herd and reported a $8.8 \%$ standard error of estimation of SM from BTSCC. In their study, the BTSCC information consisted of 52 readings per herd, summarized in the form of a yearly Cpk index. In our data set, each bulk tank that the herd shipped to the milk processor was tested for SCC resulting in from 182 to 365 readings per herd. This more complete representation of herd's everyday performance might have contributed to the greater accuracy of our model at estimating SM than equations developed by Niza-Ribeiro et al. (2004) (standard error of estimation, 4.02 vs. $8.8 \%$ ).

Capability index was designed to summarize the performance of a stable process, that is, one that does not experience any changes. The mean and standard deviation used in its calculation are representative of an average state of the process during a specific period, making the Cpk fairly insensitive to isolated changes in process performance. Our models (similar to Cpk) are based on estimates of mean and sigma, and therefore, will not reflect any single unsustained shift in the process. Single test-day SM estimates are representative of the state of the herd on one particular day and are sensitive to single-day upward or downward shifts. Therefore, an attempt to correlate the BTSCC data with single test-day SM in our data set would not yield satisfactory results and thus, a different approach was chosen. Research suggests that in the case of individual cows, an average of several test-day results is more accurate at identifying cows with high SCC (Reneau, 1986; Schepers et al., 1997). Similarly, an average of multiple test days' estimates of SM will give a better estimate of herds' SM prevalence, less sensitive to single-day variation. Therefore, the fact that the models in our study were developed based on an average of 10 or more test days across the year for $82 \%$ of the herds, increases the ability of the models to estimate the true mean SM prevalence in the herd. This also means, however, that the models are not designed to respond to unsustained changes in SM prevalence. Our models can be used in herds with no DHIA testing as a way of estimating mean SM in the herd. They can also potentially be used to estimate a new SM after a sustained shift in BTSCC or SM for a stable process (not experiencing any changes).

Consideration has to be given to the number of BTSCC readings needed to accurately estimate SM using our models. Quesenberry (1997) suggests that, to establish an individual measurement chart, a sample size of at least 100 is necessary for true mean and variation estimates of the output of a stable process; however, our models only require means of BTSCC to estimate the SM in the herd. Moreover, it is well established that BTSCC is subject to sustained seasonal changes that might partly reflect the changes of the SM in the herd. From this standpoint, an estimate of SM based on a monthly mean of BTSCC based on 15 to 30 readings should be representative of the average SM status during that month if every milk shipment is tested. On the other hand, in herds that have their milk tested only once or twice a month, yearly data would have to be used to estimate the SM in the herd.

Due to the direct and strong correlation between BTSCC and SM, plotting BTSCC daily on an SPC chart and monitoring the charts for signals of true change 
would help the dairy manager recognize shifts in SM daily without waiting for the DHIA test-day report. Exercising the use of an SPC chart to identify shifts in SM was the aim of the case study examples presented below.

\section{Case Study}

In our case study we included 5 herds, one from each herd production category. We used the $30 \mathrm{~d}$ preceding the 1 test day before the increase in SM (D1) to estimate mean and sigma and calculate the center line and upper and lower 3-sigma control limits. We plotted the BTSCC results from $30 \mathrm{~d}$ before D1 until D2 testing for special cause variation between the 2 adjacent DHIA test days marking the greatest increase in SM (D1 and D2) by applying 5 run rules. The run rules were: $\mathrm{A}=\mathrm{A}$ point outside the 3 -sigma control limits; $\mathrm{B}=9$ points in a row on one side of the center line; $\mathrm{C}=6$ points in a row, either all increasing or all decreasing; $\mathrm{D}=2$ out of 3 points in a row in zone $\mathrm{A}$ or beyond on one side of the center line; and $\mathrm{E}=4$ out of 5 points in a row in zone $\mathrm{B}$ or beyond on one side of the center line. Results are presented in Figure 3 and the description of the herds is presented in Table 1.

Once a point on the Shewhart chart met any 1 of the 5 run rules, it was considered a signal of change in process performance. All charts but one signaled a significant increase in BTSCC before D2, when DHIA test identified the increase in SM. For herd A, no point on the Shewhart chart met any of the run rules, causing the chart not to signal any change in the process. Herd A was characterized by very large variation $(181,000$ cells/mL SCC Table 1). For a given change in mean, the greater the variation, and the more time it takes a Shewhart chart to signal that change. The remaining charts signaled a change in BTSCC and, by correlation, a change in SM before the actual DHIA test day. This illustrates that the Shewhart chart of BTSCC could alert farmers to an increase in SM between the DHIA test days. An interesting example of how the chart could have helped the farmer is given by Figure 3B. The Shewhart individual measurement chart analysis suggests that since the beginning of June, the herd had been experiencing an increase in BTSCC. If the farmer had been presented with this chart, he could have identified the need to increase vigilance. In response to the signal on the chart, the farmer could have prevented the spread of mastitis by identifying the sick cows, increasing bedding changing frequency, and ensured better compliance to the proper milking procedure.

As mentioned above, during a DHIA test day all cows in milk are tested and contribute to the SM estimation. Therefore, if cows with mastitis were identified before
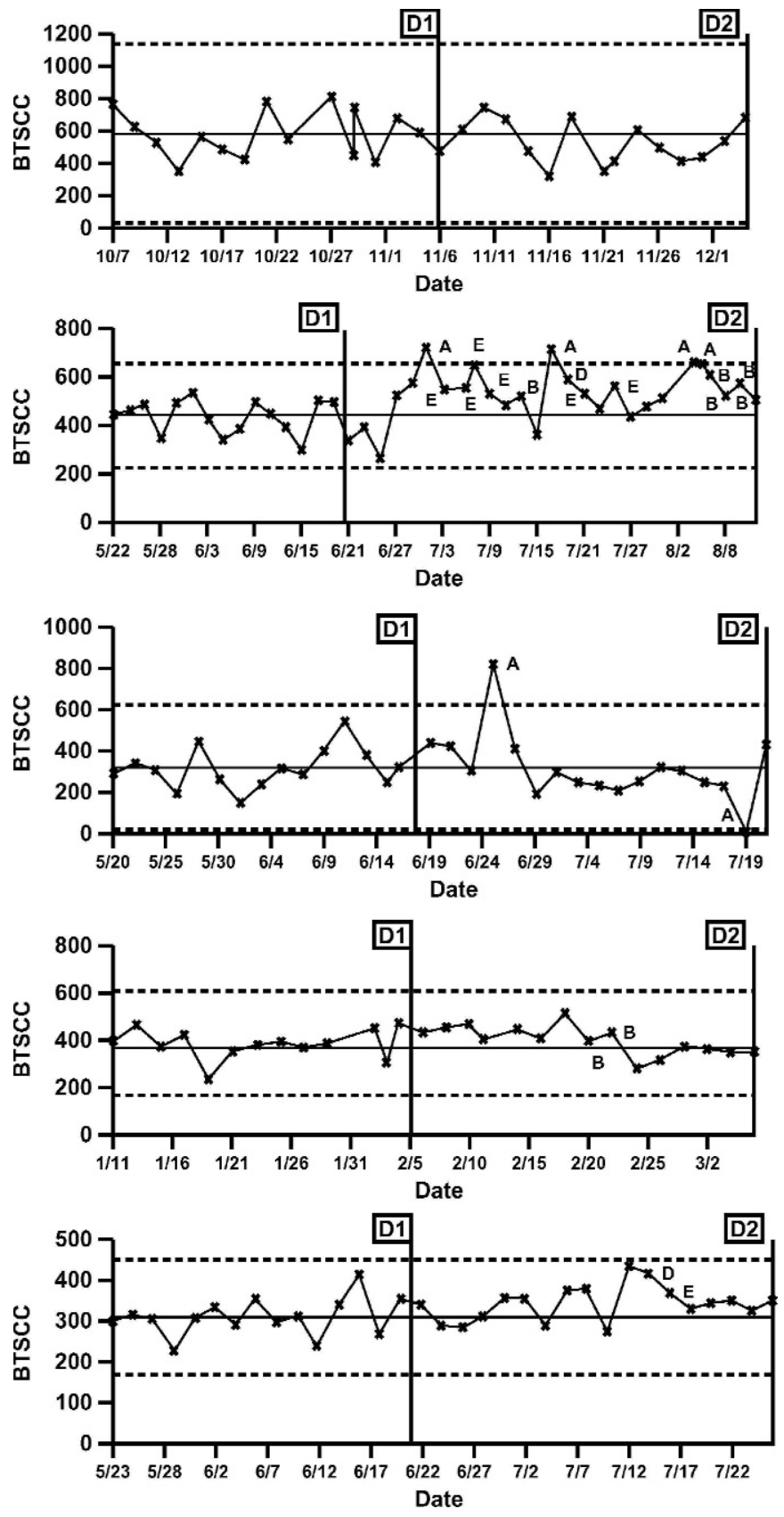

Figure 3. Individual measurement charts of the bulk tank SCC (BTSCC) results obtained between the 2 adjacent DHIA test days marking the greatest increase in subclinical mastitis prevalence (SM) (D1 and D2 marked by the horizontal lines). Upper and lower control limits (dashed lines) and the center line (solid line) were computed from mean and sigma estimates based on the bulk tank SCC from the $30 \mathrm{~d}$ preceding D1. Five run rules identifying special cause variation are coded as follows: $\mathrm{A}=\mathrm{A}$ point outside the 3 -sigma control limits; $\mathrm{B}=9$ points in a row on one side of the center line; $\mathrm{C}=6$ points in a row, either all increasing or all decreasing; $D=2$ out of 3 points in a row in zone A or beyond on one side of the center line; and $\mathrm{E}=4$ out of 5 points in a row in zone $\mathrm{B}$ or beyond on one side of the center line. 
the DHIA test day and their milk was eliminated from the bulk tank, the BTSCC would be affected. The graphs presented in Figure 3C indicate that that might have been the case for herd $\mathrm{C}$. The chart first signaled at the upper control limit indicating an increase in mean, and then signaled on the lower control limit suggesting a decrease in BTSCC before D2. However, the DHIA SM estimate on D2 indicates an increase in SM compared with D1. This suggests that although BTSCC has decreased, possibly by eliminating milk from the mastitic cows from the bulk tank, the sick cows were still in the herd.

In the case study examples, the control limits were calculated from results of a 30-d period preceding D1. This approach was chosen for 2 reasons: 1 ) to characterize current process performance (in terms of mean and variation) before the increase in subclinical mastitis prevalence in herds, and 2) to limit the effect of season on BTSCC. Further studies investigating the use of SPC charts to detect changes in mastitis prevalence from herd's BTSCC should include testing different SPC charts (Individual Measurement, Exponentially Weight Moving Average, Cumulative Sum chart) and approaches to calculating control limits (moving window, autocorrelation function approach) in terms of sensitivity and specificity.

\section{NIR}

Neither of the methods used in the study was able to yield models with satisfactory estimations of NIR based on herds BTSCC data (data not presented). The relative difficulty of developing the NIR estimation models might be partly explained by the time lag between the DHI test days in individual herds and the difference in SM and NIR dynamics. New infection rate accounts for the rotation of cows within the subclinical mastitis-infected group. This makes the NIR value a more time-sensitive measure than SM, which only identifies the percentage of herd infected with subclinical mastitis at a single time point. Therefore, an NIR value obtained from a single test day will be less representative of the actual NIR dynamics than a single SM value of the daily SM in the herd. This factor makes it even more important to succeed in developing models that would estimate NIR based on the BTSCC so that NIR estimates could be obtained more frequently than once a month. Without more frequent measures of NIR in the herds used in our data set, the effort to develop such models was unsuccessful.

A second potential problem with relating BTSCC to NIR is that the average BTSCC are likely to be determined to a greater extent by a change in the number of cows with high SCC reflected by SM, not by the rotation within the infected cow group associated with NIR. However, the increase in SCC at the beginning of a mastitis infection (monitored by the NIR) is likely to be rapid and cause increased variation in daily BTSCC, especially in smaller herds. In this context, more frequent NIR estimates and a closer focus on the variation in BTSCC might be of value.

\section{CONCLUSIONS}

It can be concluded that monitoring individual BTSCC using SPC charts will help dairy managers identify true changes in SM on a daily basis. The models developed in this study indicate that BTSCC can also be used to estimate average subclinical mastitis prevalence during the monitored period. Single DHI testday estimates of NIR, however, proved insufficient for accurately describing NIR dynamics and relating it to herd BTSCC. Future studies should try correlating BTSCC with more frequent NIR estimates based on daily individual cow SCC testing.

\section{ACKNOWLEDGMENTS}

The authors would like to acknowledge Land O'Lakes Inc. (Arden Hills, MN) for their valuable help and cooperation with this project.

\section{REFERENCES}

Belisto, J. E., A. de Vries, and R. P. Natzke. 2004. Evaluation of the DHI Hot List as a tool to reduce bulk tank somatic cell counts. J. Dairy Sci. 87(Suppl. 1):141. (Abstr.)

Cowen, P., D. Fernandez, and H. J. Barnes. 1994. Surveillance strategies for monitoring variation in animal health and productivity: The use of statistical process control in turkey industry. Kenya Vet. 18:202-204.

de Vries, A., and B. J. Conlin. 2003. Economic value of timely determination of unexpected decreases in detection of estrus using control charts. J. Dairy Sci. 86:3516-3526.

Dial, G. D., C. Duangkaew, and C. Rademacher. 1996. Statistical process control-Application to swine production. Pages 53-83 in Allen D. Leman Swine Conference Proceedings, Veterinary Outreach Programs. University of Minnesota, St. Paul.

Eberhart, R. J., L. J. Hutchinson, and S. B. Spencer. 1982. Relationship of bulk tank somatic cell counts to prevalence of intramammary infection and indices of herd production. J. Food Prot. 45:1125-1128.

Emanuelson, U., and H. Funke. 1991. Effect of mastitis yield on relationship bulk milk somatic cell count and prevalence of mastitis. J. Dairy Sci. 74:2479-2483.

Fernandez, D. V. 1995. Determinants of productivity in commercial tom turkey production. Ph.D. Thesis, N. Carolina Univ., Raleigh.

Harmon, R. J. 1994. Physiology of mastitis and factors affecting somatic cell counts. J. Dairy Sci. 77:2103-2112.

Ishikawa, K. 1984. What is total quality control? The Japanese way. Prentice-Hall, Inc., Englewood Cliffs, NJ.

Kinsel, M. L. 2004. Understanding variation: Key to reducing BTSCC. Pages 41-44 in Natl. Mastitis Council, 2004 Regional Meeting Proceedings, Bloomington, MN. Natl. Mastitis Council, Inc., Madison, WI. 
Marsh, W. E., A. de Vries, J. K. Reneau, and M. L. Kinsel. 1997. Monitoring performance: Statistical process control in dairy herd management. Pages 34-46 in Annual Northeast Dairy Production Medicine Symposium. 6th ed. Syracuse, NY. NEDPMS, Syraccuse, NY.

Niza-Ribeiro, J., J. P. T. M. Noordhuizen, and J. C. Menezes. 2004. Capability index-A statistical process control tool to aid in udder health control in dairy herds. J. Dairy Sci. 87:2459-2467.

Norman, H. D., R. H. Miller, J. R. Wright, and G. R. Wiggans. 2000. Herd and state means for somatic cell count from Dairy Herd Improvement. J. Dairy Sci. 83:2782-2788.

Quesenberry, C. P. 1997. SPC methods for quality improvement. John Wiley and Sons, New York, NY.

Reneau, J. K. 1986. Effective use of Dairy Herd Improvement somatic cell counts in mastitis control. J. Dairy Sci. 69:1708-1720.
Reneau, J. K., and M. L. Kinsel. 2001. Record systems and herd monitoring in production-oriented health and management programs in food producing animals. Chapter 3 in Herd Health. $\mathrm{O}$. Radostits, ed. W. B. Saunders Company, Philadelphia, PA.

Sard, D. M. 1979. Dealing with data: The practical use of numerical information-(14) Monitoring changes. Vet. Rec. 105:323-328.

Schepers, A. J., T. J. G. M. Lam, Y. H. Schukken, J. M. B. Wilmink, and W. J. A. Hanekamp. 1997. Estimation of variance components for somatic cell counts to determine thresholds for uninfected quarters. J. Dairy Sci. 80:1833-1840.

USDA. 2003. 2002 Dairy Producer Opinion Survey. Minnesota Agricultural Statistics Service, St. Paul, MN.

Wiggans, G. R. 2004. USDA Summary of DHI Participation (DHI Report K-1). Online. Available http://aipl.arsusda.gov/publish/ dhi/current/partx.html. Accessed Dec. 7, 2004. 